\title{
Análisis de la variación y predicción de radiación solar en la zona de Urbina, usando la teoría del caos
}

DOI: https://doi.org/10.33262/ap.v3i3.1.101

(c) (1) (ㅇ) (9)
Analysis of the variation and prediction of solar radiation in the Urbina area, using the theory of chaos

Nelly Patricia Perugachi Cahueñas. ${ }^{1}$, Jorge Milton Lara Sinaluisa. ${ }^{2} \&$ Arquímides Xavier Haro Velasteguí. ${ }^{3}$

\begin{abstract}
.
DOI: https://doi.org/10.32/cienciadigital.v3i1.947

The variation of incident radiation in the Urbina zone (UTM x754579, y9835357; 3646 masl), located in the Altoandino paramo region of the Chimborazo province, is analyzed in order to know its dynamics and the effects of the geographical position and height with respect to at sea level, using the Chaos Theory. To control the anomalous data, the simple non-linear noise reduction method was applied; Then, a multidimensional phase reconstruction was carried out in space, determining the Lyapunov coefficients, entropy and fractal dimension of the system, allowing its dynamics and variation over time to be described. It is shown to be a chaotic (non-linear) system, by presenting more than one positive Lyapunov coefficient. In addition, the data are predicted over time and it is verified that there are no significant differences between the measured and predicted series, using the Bootstrap method.
\end{abstract}

Keywords: Solar radiation, chaos, Lyapunov, fractal, entropy.

\footnotetext{
${ }^{1}$.Escuela Superior Politécnica de Chimborazo, Facultad de Administración de Empresas, Escuela de Finansas, Chimborazo, ncperugachi@yahoo.com, https://orcid.org/0000-0001-6331-9551

2 Escuela Superior Politécnica de Chimborazo, Facultad de Administración de Empresas, Escuela de Transporte, Chimborazo, j_lara@espoch.edu.ec, https://orcid.org/0000-0002-3116-5161

${ }^{3}$ Escuela Superior Politécnica de Chimborazo, Facultad de Ciencias, Escuela de Física, Chimborazo, aharo@espoch.edu.ec, https://orcid.org/0000-0003-3391-5082
} 


\section{Resumen.}

Se analiza la variación de la radiación incidente en la zona de Urbina (UTM x754579,y9835357; $3646 \mathrm{msnm}$ ), ubicada en la región del páramo Altoandino de la provincia de Chimborazo, con la finalidad de conocer su dinámica y los efectos de la posición geográfica y altura respecto al nivel del mar, usando la Teoría del Caos. Para controlar los datos anómalos se aplicó el método de reducción del ruido no lineal simple; luego se procedió a realizar una reconstrucción en el espacio de las fases multidimensional, determinando los coeficientes de Lyapunov, entropía y dimensión fractal del sistema, permitiendo describir su dinámica y la variación en el tiempo. Se demuestra que es un sistema caótico (no lineal), al presentar más de un coeficiente positivo de Lyapunov. Además, se predicen los datos en el tiempo y se verifica que no hay diferencias significativas entre las series medidas y predichas, usando el método Bootstrap.

Palabras claves: Radiación solar, caos, Lyapunov, fractal, entropía.

\section{Introducción.}

El Sol es la principal fuente de energía para todos los procesos naturales que tienen lugar en el planeta Tierra. Hasta la Tierra llega una potencia de radiación equivalente a 1,7×10 14 $\mathrm{kW}$, lo que representa la potencia correspondiente a 170 millones de reactores nucleares de $1000 \mathrm{MW}$ de potencia eléctrica unitaria, o lo que es lo mismo, 10000 veces el consumo energético mundial (Cañada 1997, Pareja 2010).

Los rayos solares se propagan a través del espacio en forma de ondas electromagnéticas. Este fenómeno físico, más conocido como radiación solar, es el responsable de que el planeta Tierra reciba un aporte energético continuo de aproximadamente $1367 \mathrm{~W} / \mathrm{m}^{2}$. Un valor que recibe el nombre de constante solar y que, al cabo de un año, equivaldría a 20 veces la energía almacenada en todas las reservas de combustibles fósiles del mundo (petróleo, carbón, entre otros etc.) (Escudero 2017).

Para establecer con exactitud la cantidad de energía solar que se puede aprovechar, se deberá tener en cuenta varios aspectos como: la hora del día, la estación del año, latitud, longitud y especialmente las condiciones atmosféricas (Reyes 2001).

La contribución de la energía solar térmica al consumo mundial, sigue siendo escasa, pese a que empiezan a percibirse ciertos síntomas de cambio con miras al futuro. Al creciente interés de los ciudadanos por este tipo de soluciones hay que sumar las ayudas e incentivos que se han puesto en marcha en muchos países del mundo y la reducción de precios de los captadores solares en algunos mercados, situación que pone de manifiesto, la presencia de una tecnología madura que ha experimentado un significativo avance durante los últimos años (Pareja 2010). 
En la década de 1970 fue cuando más se desarrolló la Teoría del Caos. En 1971 David Ruelle y Takias propusieron una nueva teoría para fluidos turbulentos basada en un atractor extraño. Años, luego May encontró ejemplos del caso en mapas de aumento de población. Y a continuación vino el más sorprendente descubrimiento de todos de la mano de Feigenbaum. Él descubrió que hay leyes universales concretas que diferencian la transición entre el comportamiento regular y el caos (Ruelle 2013).

La Teoría del Caos se ha convertido en una de las herramientas más efectivas para estudiar sistemas conocidos como complejos o caóticos, como son aquellos atmosféricos, en los cuales la estadística tradicional no ha dado buenos resultados, razón por la que fue necesario buscar alternativas que permita describir este tipo de sistema con mayor precisión (Kantz 2004, Hegger 2007).

En los sistemas reales es frecuente encontrar señales que aparentemente tienen un comportamiento no casual, caracterizado por una elevada sensibilidad a las condiciones iniciales e imprevisibilidad a través del tiempo. Este tipo de sistemas se definen como caóticos, los cuales se caracterizan a través de ciertas variables en el espacio de las fases donde las dimensiones representan variables dinámicas, figura 1 (Martins 2011, Taher 2016).

Figura 1.

Representación de una hiper-esfera en el espacio de las fases, con tres dimensiones (X).

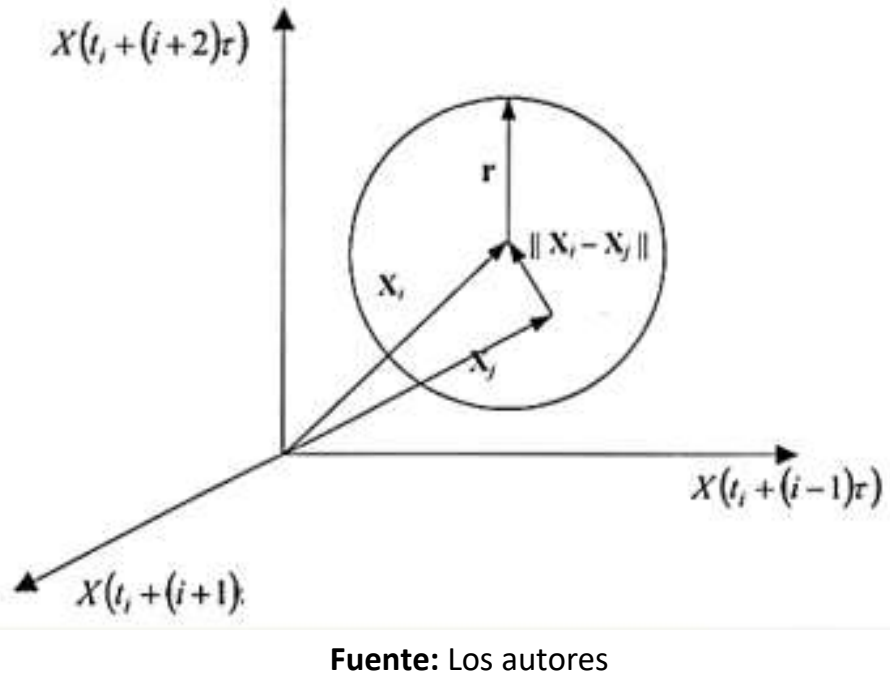

El presente estudio se propone analizar la variación de los datos de radiación global y difusa de la estación meteorológica de Urbina, perteneciente al Grupo de Energía Alternativa y Ambiente, de la Escuela Superior Politécnica de Chimborazo, la cual viene recolectando datos regularmente desde el 2014, para lo cual se propone usar la Teoría del Caos, considerando las características de las variables, posición geográfica y altura respecto al nivel del mar, dicha variación será del tipo caótica. Por la sensibilidad que presentan estas zonas para la conservación ambiental y en vista que no se han hecho estudios de este tipo en estas condiciones se plantea el presente estudio que permita conocer más a fondo su dinámica. 


\section{Metodologia.}

\subsection{Caracterización de datos usando la Teoría del Caos}

Los datos de una serie pueden caracterizarse usando la Teoría del Caos, iniciando con la reconstrucción de las series en el espacio de las fases, para lo cual se debe determinar el tiempo de retardo y la dimensión de encaje, las cuales permiten representar dichos datos en un espacio multidimensional, en el cual se estudian sus propiedades dinámicas como la dimensión fractal y la entropía del sistema, siendo los coeficientes de Lyapunov uno de los indicadores más importantes del sistema, con los que se determina si el sistema es caótico o no, también se puede predecir el comportamiento futuro de los datos, para lo cual se usa el paquete informático TISIAN (Martins 2011, Taher 2016).

\subsection{Reconstrucción de una serie de tiempo en el espacio de las fases}

Una serie de tiempo puede considerarse como una secuencia de observaciones $\left\{S_{n}=S\left(x_{n}\right)\right\}$. Puesto que la secuencia (generalmente escalar) $\left\{S_{n}\right\}$ en sí misma no representa adecuadamente el espacio de fase (multidimensional) de los sistemas dinámicos, se tiene que emplear alguna técnica para determinar la estructura multidimensional utilizando los datos disponibles (Sauer 1991). La técnica de reconstrucción de espacio de fase más importante es el método de retardo. Vectores en un espacio nuevo, el espacio de encaje, se forman valores de la serie de tiempo retrasados obteniendo la matriz de la ecuación 1.

$$
S_{n}=\left(S_{n-(m-1)} \tau, S_{n-(m-2)} \tau, \ldots, S_{n}\right)
$$

$\mathrm{S}_{\mathrm{n}}$ - matriz reconstruida de la serie de datos, $\tau$ tiempo de retardo, $m$ dimensión de encaje, valores que serán calculados con los métodos a proponer.

\subsection{Tiempo de retardo}

Para determinar un tiempo de retardo $(\tau)$ razonable, que es un valor entero adimensional que permite reconstruir la serie como matriz para ser representado en un espacio multidimensional, se usa el método de información mutua dada por Fraser y Swinney en 1986. A diferencia de la función de autocorrelación (Gallego 2010), la información mutua tiene en cuenta también la función no lineal, calculada con la ecuación 2.

$$
S_{m}=-\sum_{i j} p_{i j}(\tau) \ln \frac{p_{i j}(\tau)}{p_{i} p_{j}}
$$

Donde: $S_{m}$ función de información mutua, $p_{i}$ es la probabilidad de encontrar un valor de la serie temporal en el intervali-ésimo, y $p_{i j}$ es la probabilidad conjunta de que una observación se encuentre en el i-ésimo intervalo y más tarde j-ésimo, con $i$ y $j$ que varían según el número de datos. En teoría, esta expresión puede calcularse fácilmente si el valor de retardo $\tau$ encuentra un mínimo, el mismo es una buena opción para usarlo como tiempo de retardo, en general se asume el primer mínimo de la ecuación 2 como un valor aceptable del tiempo de retardo, Fig. 2 (Ivancevic 2007). 
Figura 2.

Determinación del tiempo de retardo usando el método de información mutua.

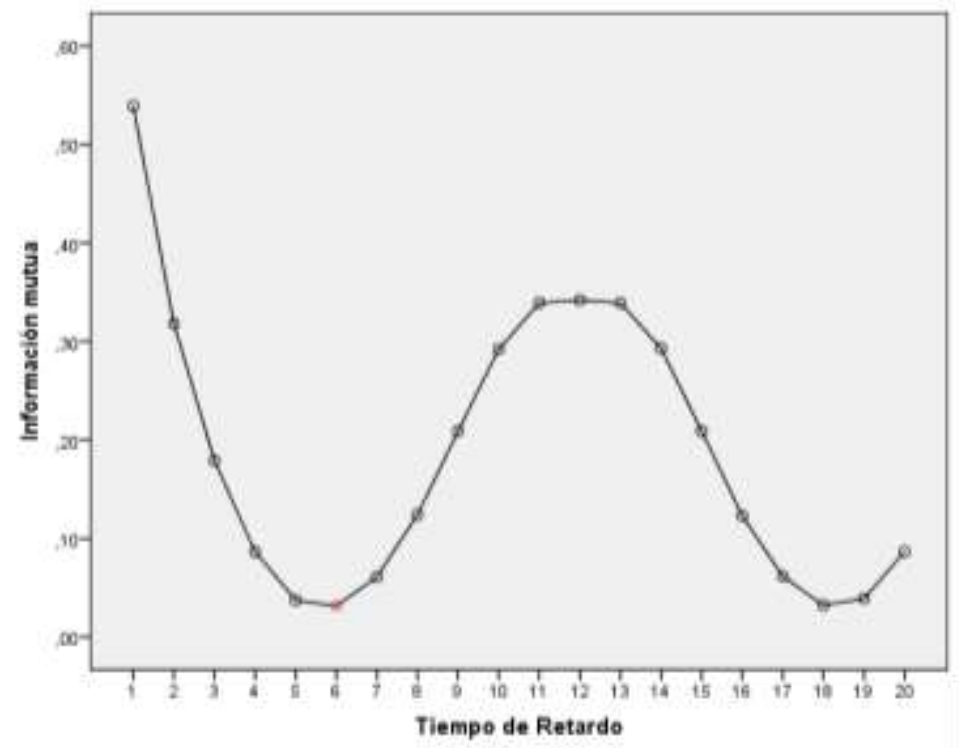

Fuente: Los autores

\subsection{Dimensión de encaje (Embedding)}

El número $m$ de elementos se denomina la dimensión de encaje, el teorema de Takens (Ruelle 2013, Haro 2016) establece que si $\left\{S_{n}\right\}$ es la sucesión de mediciones de un sistema dinámico; entonces, la dimensión de encaje con tiempo de retardo adecuado, proporciona una imagen unívoca del conjunto original, si $m$ es lo suficientemente grande.

La dimensión de encaje es muy utilizada, la aplicación es sencilla, si se dispone de mediciones escalares $N$, se construye un conjunto de vectores de encaje $m$ con dimensión $N-\tau(m-1)$. Estos deben tenerse en cuenta para el cálculo de cantidades promedios en el espacio de las fases. Hay literatura sobre la elección de los parámetros $m$ óptimos. Se propone un método para determinar la mínima dimensión $m$, donde se acepta la dimensión cuando la función es cero (Ivancevic 2007, Fernández 2014).

\section{Figura 3.}

Representación en el espacio de los falsos vecinos, proyectados en dos y tres dimensiones.
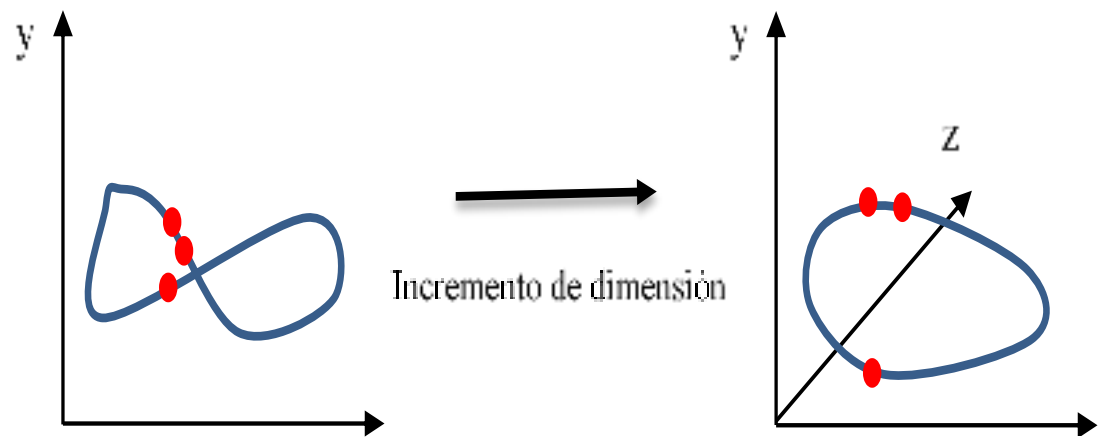

$\mathrm{x}$

Fuente: Los autores 
La idea es que por cada punto en la serie de tiempo se busquen los vecinos más cercanos $\vec{s}_{j}$ en un espacio $m$-dimensional. Calculando la distancia $\left\|\vec{s}_{i}-\vec{s}_{j}\right\|$ entre los puntos y calculando la razón dado en la ecuación 3 (Haro 2016):

$$
R_{i}=\frac{\left\|\vec{s}_{i+1}-\vec{s}_{j+1}\right\|}{\left\|\vec{s}_{i}-\vec{s}_{j}\right\|}
$$

Si $R_{i}$ excede un valor umbral $R_{t}$ este punto es llamado un falso vecino, figura 3 , el criterio es que la dimensión se acepta, cuando la fracción de los puntos, por $R_{i}>R_{t}$ es lo suficientemente pequeña o cero.

\subsection{Reducción simple del ruido no lineal}

Para implementar este esquema de reducción de ruido, primero hay que elegir una dimensión $m$, también es conveniente elegir un tiempo de retardo. A continuación, para cada vector de encaje, se forma una vecindad en el espacio de fase que contiene todos los puntos $\{\mathrm{Sn}\}$, un vecino $\mu_{\varepsilon}^{(n)}$, se forma en el espacio de las fases conteniendo los puntos $\left\{S_{n^{\prime}}\right\}$ como $\left\|S_{n}-S_{n^{\prime}}\right\|<\varepsilon$. El valor del radio de los vecinos $\varepsilon$, debe tomarse lo suficientemente grande para cubrir la extensión de ruido, pero más pequeño que un radio de curvatura típico. Estas condiciones no siempre se pueden cumplir simultáneamente, en cuyo caso hay que evaluar y repetir el proceso varias veces. Para cada vector de embending $S_{n}=\left(S_{n-(m-1)}, \ldots, S_{n}\right)$, se calcula una coordenada media corregida $S_{n-m / 2}$ promediando sobre la vecindad $\mu_{\varepsilon}^{(n)}$ (Fernández 2014), Ecuación (4):

$$
\hat{\delta}_{n-m / 2}=\frac{1}{\left|\mu_{\varepsilon}^{(n)}\right|} \sum_{\delta_{n^{\prime}} \in \mu_{\varepsilon}^{(n)}} \delta_{n^{\prime}-m / 2}
$$

\subsection{Dinámica de un sistema caótico}

El caos surge del crecimiento exponencial de las perturbaciones infinitesimales, para garantizar el análisis de esta inestabilidad están los exponentes de Lyapunov, que miden cuánto se alejan entre dos trayectorias, ecuación 5 (Constantin 2006).

$$
\lambda_{i}=\frac{1}{t} \ln \left(\frac{r_{i}(t)}{r}\right)
$$

Con $r_{i}(t)$ semieje mayor de una elipse, $r$ radio pequeño inicial y $\mathrm{t}$ tiempo grande, en general se establece:

$\lambda_{1} \geq \lambda_{2} \cdots \geq \lambda_{d}$

Variables que permiten caracterizar un atractor, así:

- Para un punto fijo, todos $\operatorname{los} \lambda_{i}$ son negativos.

- $\quad$ En un ciclo límite, $\lambda_{1}=0$ y $\lambda_{i}<0$ con $i>1$.

- En un sistema caótico al menos un exponente de Lyapunov es positivo. 
A partir de este resultado se puede definir, la entropía de Kolmogorov - Sinai (Garín 2015), ecuación (6).

$$
h=\sum_{i=\# \lambda>0} \lambda_{i}
$$

O la dimensión de Kaplan - Yorke, 1979, ecuación 7.

$$
D_{F}=j+\frac{\sum_{i=1}^{j} \lambda_{i}}{\left|\lambda_{j+1}\right|}, \operatorname{con} \sum_{i=1}^{j} \lambda_{i}<0
$$

Con $\mathrm{j}$ - coeficientes positivos de Lyapunov (Constantin 2006).

\subsection{Predicción de la serie de tiempo usando la Teoría del Caos}

Usando los parámetros determinados que caracterizan el espacio de las fases para un determinado valor de la serie de datos $x(t)$, es posible hacer modelos locales para describir la evolución desde una vecindad (con verdaderos vecinos) hacia otra vecindad de la órbita $x(t+1)$ posterior. En este caso se ha usado la función Gaussiana, con puntos alrededor de los elegidos para reproducir los datos de la serie de tiempo. La varianza de la función Gaussiana se fija a la distancia media entre los centros. El modelo predice la serie de datos mediante la ecuación 8:

$$
X_{n+1}=a_{0}+\sum a_{i} f_{i}\left(x_{n}\right)
$$

Donde $x_{n}$ es el vector de n-ésimo retardo y $f_{i}$ es una función gaussiana centrada en el punto i-ésimo y $a$ constantes. Lo cual se puede aplicar a los parámetros atmosféricos de ingreso del secador solar para predecir su comportamiento y simular en función del tiempo el funcionamiento del modelo propuesto (Medvinsky 2017, Domański 2020).

\subsection{Método Bootstrap}

Los métodos bootstrap son una clase de métodos Monte Carlo no paramétricos que pretenden estimar la distribución de una población mediante remuestreo. Los métodos de remuestreo tratan una muestra observada como una población finita, y generan muestras aleatorias a partir de ella para estimar características poblacionales y hacer inferencia sobre la población muestreada. Una de las problemáticas que ataca este tipo de método es la estimación de intervalos de confianza o diferencia de medias (Zuñiga 2017)

\subsection{Fundamentos matemáticos de la correlación}

El error cuadrático medio se define por:

$$
E C M=\frac{\sum_{i=1}^{n}\left(e_{i}\right)^{2}}{n}
$$

Siendo: $e_{i}$ la diferencia para cada elemento entre la observación real y el dato estimado Dato real versus dato estimado, y n el número de elementos observados. 
La raíz de este error nos da el equivalente a su desviación típica:

$$
R E C M=\sqrt{\frac{\sum_{i=1}^{n}\left(e_{i}\right)^{2}}{n}}
$$

De esta surge el Coeficiente de Correlación de Pearson, el más conocido de los coeficientes de correlación:

$$
R=\frac{\sigma_{x y}}{\sigma_{x} \sigma_{y}}
$$

Siendo: $\sigma_{x y}$ la covarianza de las variables $\mathrm{X}$ e $\mathrm{Y}, \sigma_{\mathrm{x}}$ la desviación típica de la variable $\mathrm{X}$, y $\sigma_{\mathrm{y}}$ la desviación típica de la variable $\mathrm{Y}$, para valores entre 0 y 1 se considera de alta correlación (Puente 2018).

\subsection{Zona de estudio y equipos usados}

El estudio se desarrolla en el páramo de Urbina en la Provincia de Chimborazo a 3646 metros sobre el nivel del mar en la estación meteorológica automática tipo Vaisala, de la Escuela Superior Politécnica de Chimborazo que maneje el Grupo de Energía Alternativa y Ambiente (GEAA) de la Facultad de Ciencias, ubicado en las coordenadas UTM x754579 y9835357; de la cual se ha tomado los datos de radiación difusa y global con sensores de radiación tipo SR11 desing, entre los años 2014 al 2019, en intervalos de una hora de tiempo.

Figura 4.

Ubicación de la estación meteorológica de Urbina, del grupo GEAA de la ESPOCH.

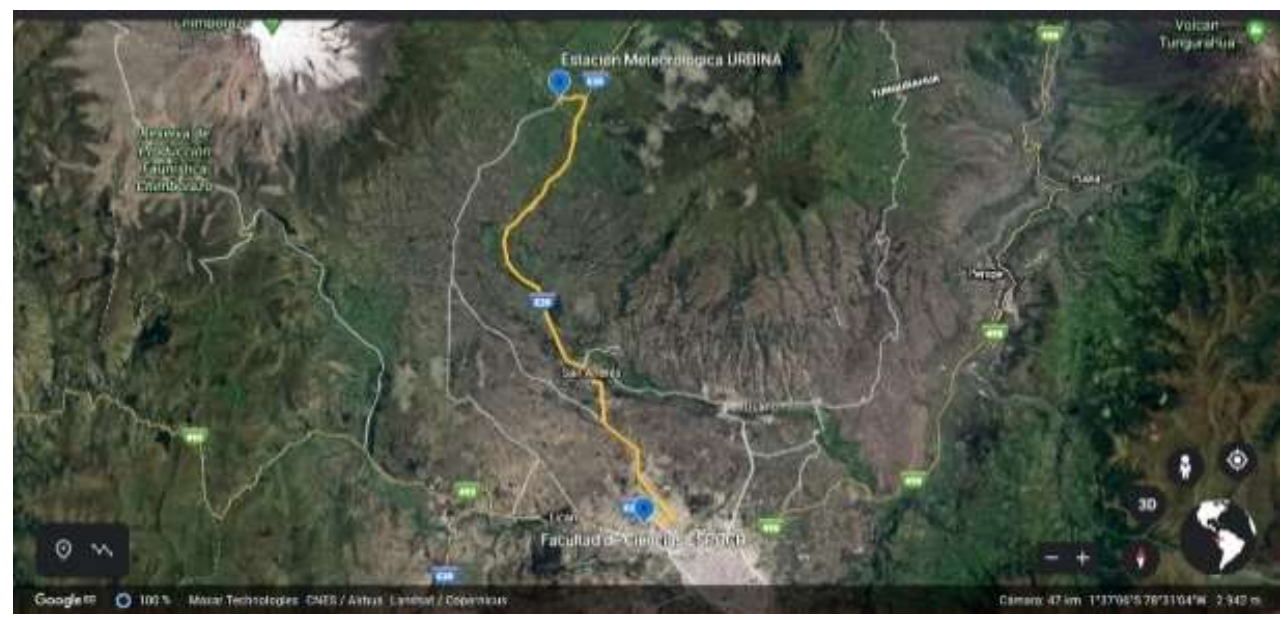

Fuente. Imagen Google.

\section{Resultados.}

En la Tabla 1 se observan los resultados estadísticos de la radiación global y difusa registrados en la estación meteorológica de Urbina; también se presenta un cálculo realizado con la ecuación 13 de las horas teóricas de sol que debería haber dada la posición de la zona, y las horas efectivas de radiación calculadas según la Organización Mundial 
de Meteorología (OMM), que establece como radiación efectiva aquellas que superan los $120 \mathrm{~W} / \mathrm{m}^{2}$.

$$
h_{e}=\left(\frac{2}{15}\right) \operatorname{arcos}(\tan \varphi \cdot \tan \lambda)
$$

Con $h_{e}$ horas efectivas de sol, $\varphi$ declinación solar y $\lambda$ latitud.

Tabla 1.

Análisis estadístico de los datos medidos de radiación difusa y radiación global de la estación meteorológica de la ESPOCH de la zona de Urbina.

\begin{tabular}{lrr}
\hline \multicolumn{1}{c}{ Variables estadísticas } & Radiación Difusa & Radiación Global \\
\hline Horas de sol teóricas $(\mathrm{h})$ & 12 & 12 \\
Horas de sol efectivas $(\mathrm{h})$ & 8,5 & 9,1 \\
Media $\left(\mathrm{W} / \mathrm{m}^{2}\right)$ & 142,83 & 181,46 \\
Moda $\left(\mathrm{W} / \mathrm{m}^{2}\right)$ & 0 & 0 \\
Coeficiente de asimetría & 1,87 & 1,53 \\
Desviación estándar $\left(\mathrm{W} / \mathrm{m}^{2}\right)$ & 215,48 & 267,25 \\
Mínimo $\left(\mathrm{W} / \mathrm{m}^{2}\right)$ & 0 & 0 \\
Máximo $\left(\mathrm{W} / \mathrm{m}^{2}\right)$ & 1259,18 & 1298,22 \\
\hline
\end{tabular}

De los resultados presentados en la Tabla 1, se establecen 12 horas de sol teóricas en la zona y una radiación efectiva de 8,5 horas para la radiación difusa y 9,1 en la radiación global, resultado que se da normalmente a partir de las 8:00 hasta las 17:00 horas. Además, se observa una media de $142,83 \mathrm{~W} / \mathrm{m}^{2}$ para la radiación difusa y $181,46 \mathrm{~W} / \mathrm{m}^{2}$ en la radiación global; considerando una moda de cero que se da en el anochecer, con coeficientes de asimetría de 1,87 y 1,53 respectivamente para la radiación difusa y global, es claro que existe una distribución asimétrica positiva, valores máximos de radiación alcanzan valores de $1298,22 \mathrm{~W} / \mathrm{m}^{2}$ en radiación global y $1259,18 \mathrm{~W} / \mathrm{m}^{2}$ en la difusa, desviación estándar de 215,48 y 267,25 W/m² respectivamente para radiación solar difusa y global.

Figura 5.

Gráfico de datos de radiación solar difusa con reducción de ruido.

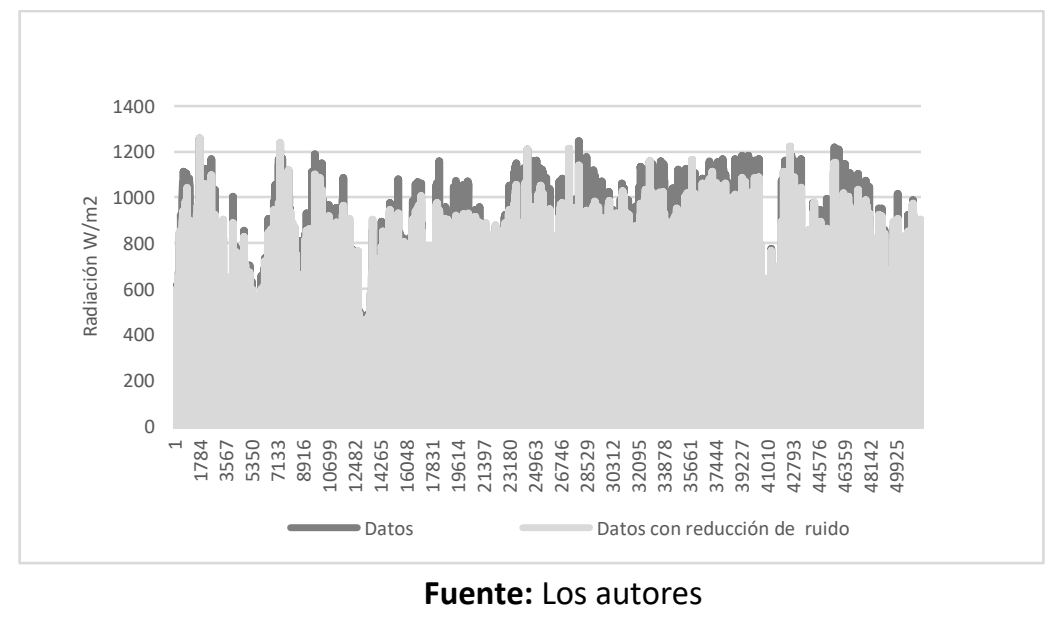


Figura 6.

Gráfico de datos de radiación solar global con reducción de ruido.

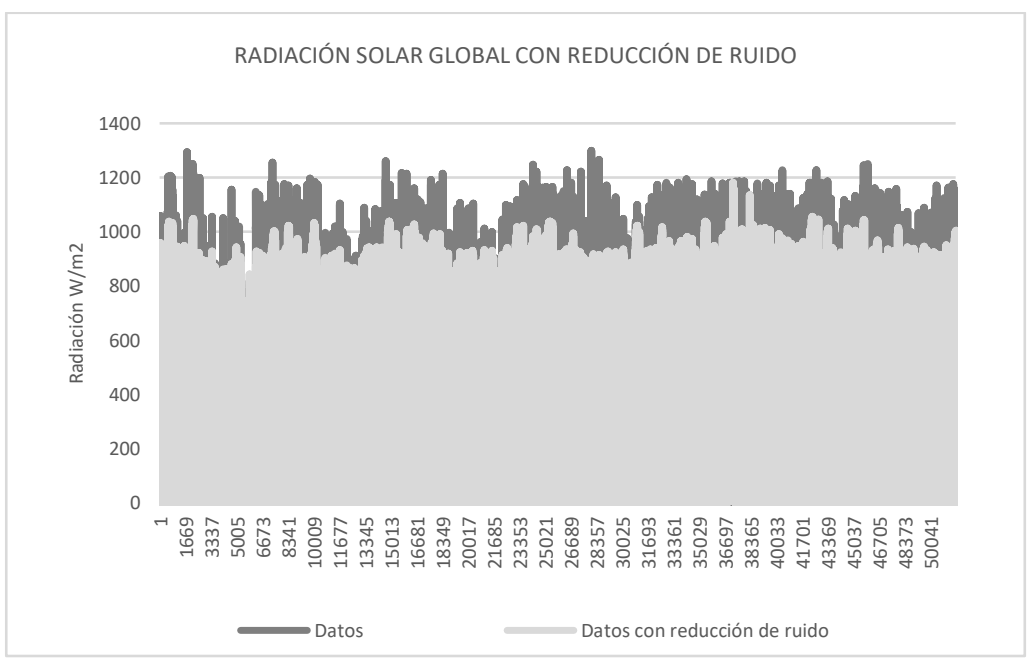

Fuente: Los autores

En el procesamiento de datos usando la Teoría del Caos, para reducir los anómalos se hace la reducción del ruido; cuyos resultados que se presentan en las figuras 5 y 6 , notándose mayor irregularidad en la radiación solar global, lo que implica más reducción del ruido; procedimiento que nos da una base de datos depurada y que es recomendado realizar cuando los son muy irregulares.

Tabla 2.

Tiempo de retardo (delay time) y dimensión de encaje (ebedding dimention) con reducción del ruido.

\section{PARÁMETERO TIEMPO DE RETARDO DIMENSIÓN DE ENCAJE}

\begin{tabular}{lll} 
Radiación Dirécta & 6 & 20 \\
Radiación Global & 6 & 20 \\
\hline
\end{tabular}

Para realizar la reconstrucción de los datos en el espacio de las fases, se procede a calcular el tiempo de retardo y la dimensión de encaje con los métodos propuestos, tanto en la radiación solar global como en la difusa. Los resultados presentan valores similares entre las dos series; lo que indica la similitud en el comportamiento de las mismas, lo cual se puede apreciar en la Tabla 2. 
Figura 7.

Gráfico de datos los coeficientes de Lyapunov calculados de la radiación solar difusa y global.

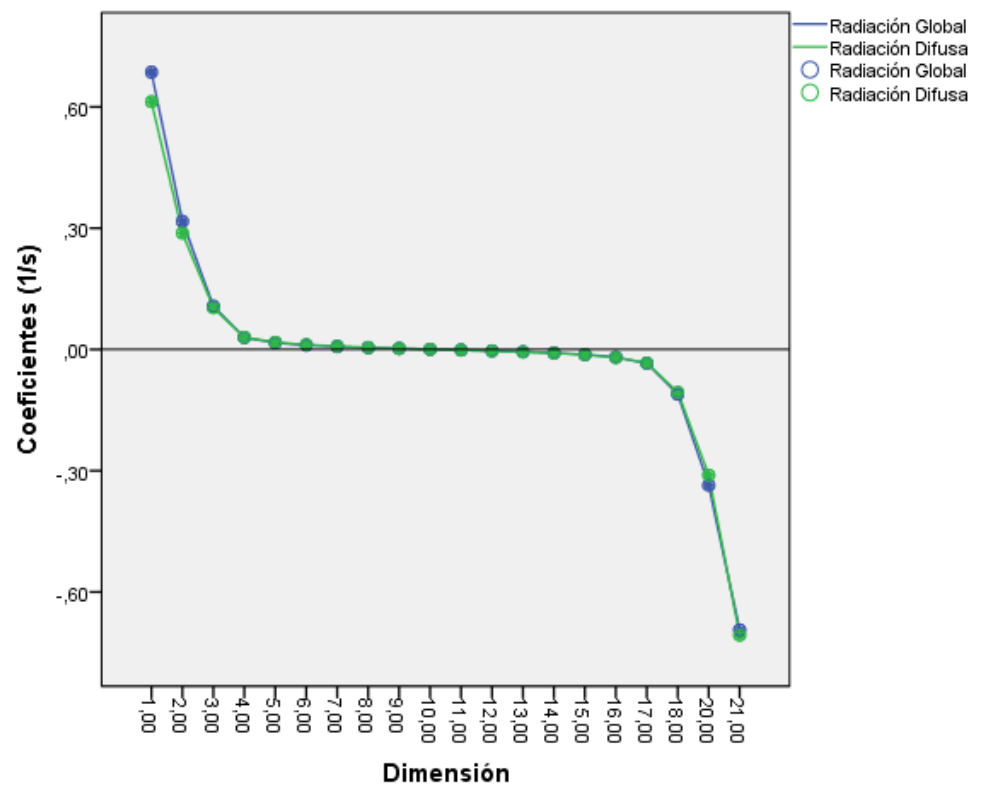

Fuente: Los autores

El espectro de los coeficientes de Lyapunov en los dos sistemas, es bastante similar como se puede observar en la figura 7, alcanzando 10 coeficientes positivos de Lyapunov, que demuestra la alta irregularidad en el comportamiento de los datos, siendo los de mayor significancia son los tres primeros.

Tabla 3.

Dimensión Fractal y entropía del sistema.

\begin{tabular}{lcc}
\hline & $\begin{array}{c}\text { DIMENSION } \\
\text { FRACTAL }\end{array}$ & ENTROPYA \\
\hline Radiación Difusa & 19,81 & $1,08 \mathrm{E}+00$ \\
Radiación Global & 19,93 & $1,18 \mathrm{E}+00$ \\
\hline
\end{tabular}

Se determina la dimensión fractal (Tabla 3), que en cada caso es mayor a 19, lo cual indica la dimensión mínima del sistema en el espacio de las fases. Además, la dimensión es fraccionaria, presentando una geometría fractal, lo que indica la complejidad del sistema; de igual manera se halla la entropía que mide el grado de irregularidad del sistema, que impide su predictibilidad con exactitud, siendo un poco más alto en la radiación global, lo que se pudo inducir anteriormente al reducir el ruido.

Con los resultados se procede a realizar la predicción de la radiación global y difusa; lo que permitirá simular el sistema; se ha realizado un análisis comparativo con los datos reales, para establecer la precisión de la misma. 
Figura 8.

Gráfico de las series de datos predichos y reales de la radiación difusa usando la Teoría del Caos.

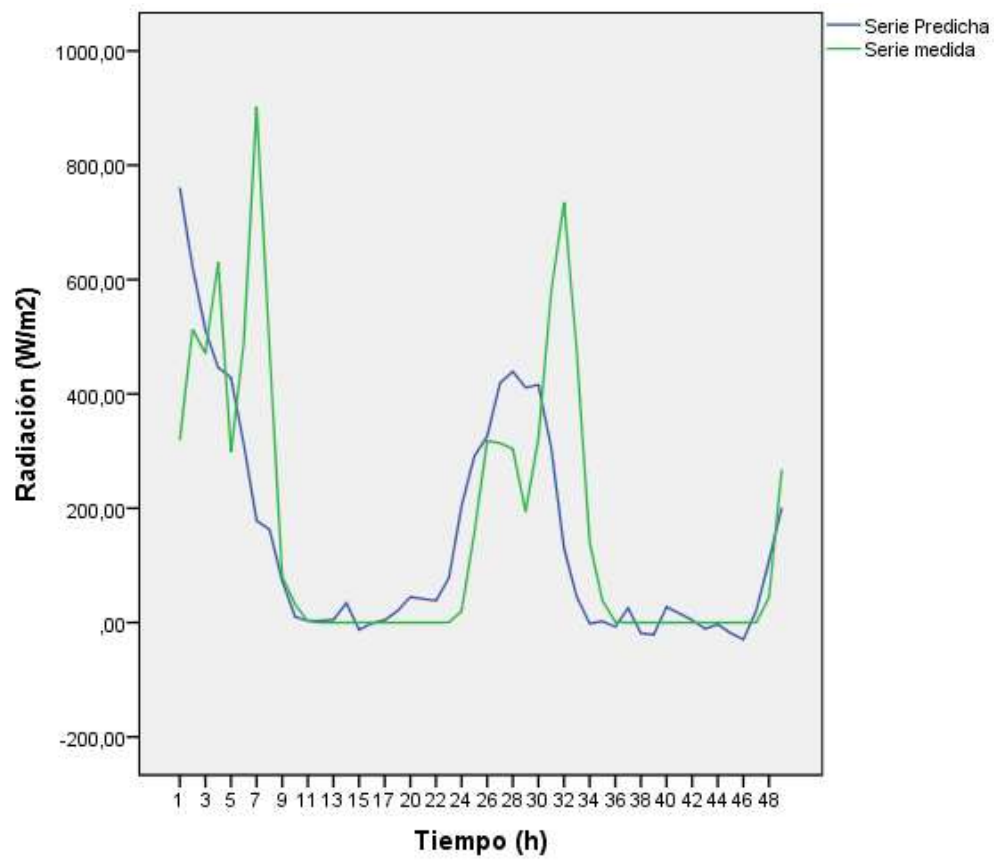

Fuente: Los autores

Figura 9.

Gráfico de las series de datos predichos y reales de la radiación global usando la Teoría del Caos.

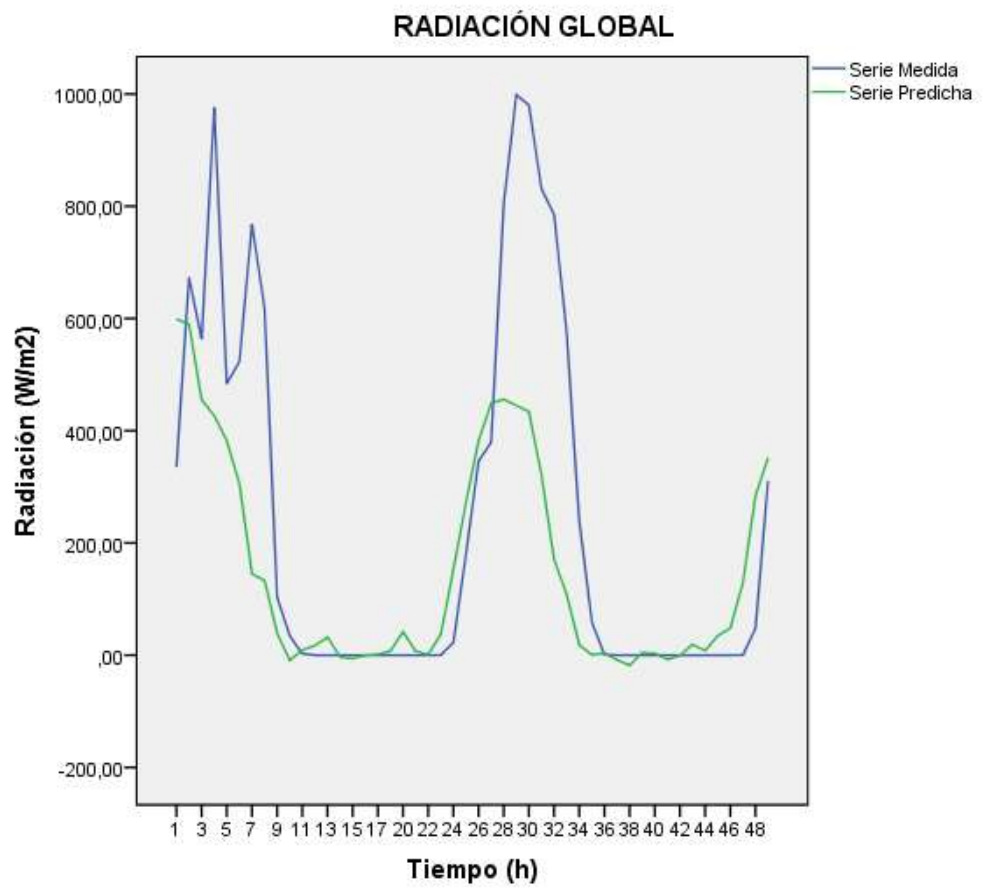

Fuente: Los autores

Las figuras 8 y 9 se muestran los resultados de las radiaciones predichas y las reales. En la figura 10 se presenta un analisis de diferencia de medias usando el método Boostrap al 0,5 de significancia, determinándose que no hay diferencias significativas entre las series. 
Figura 10.

Prueba de hipótesis usando el método Bootstrap entre las series predichas con la Teoría del Caos para las series de radiación difusa y global.
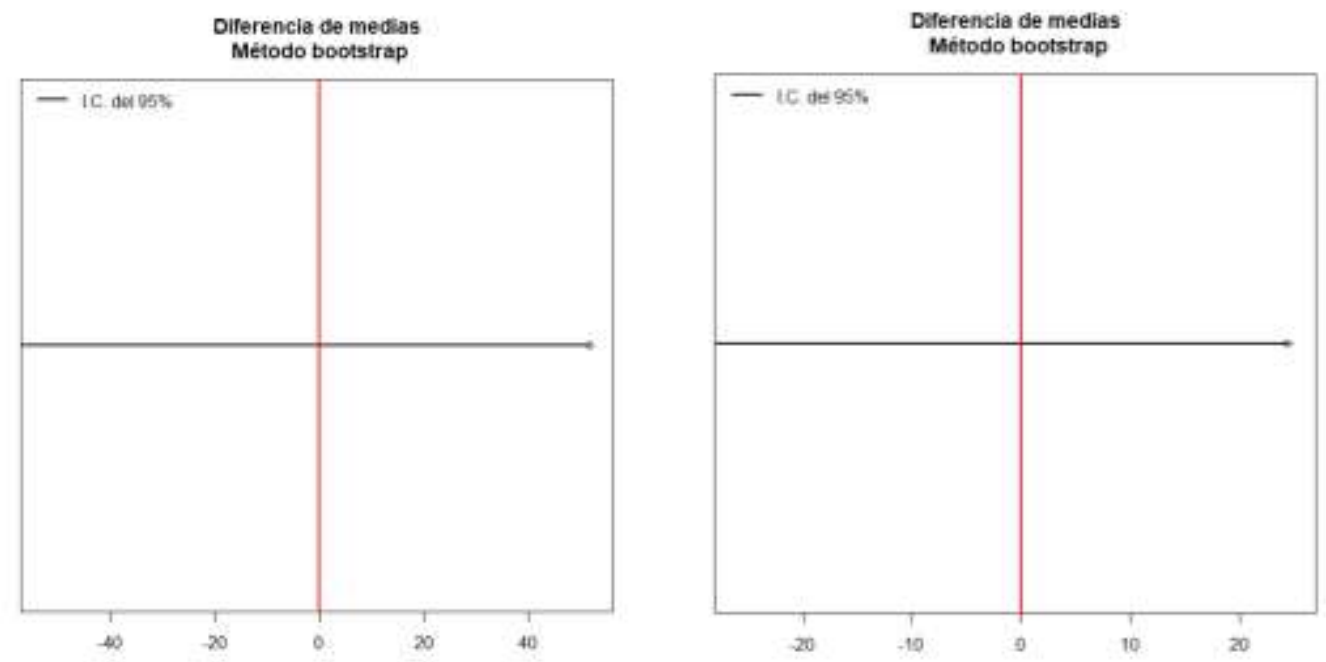

Fuente: Los autores

Tabla 4.

Error cuadrático medio entre series de datos reales con predichos de la radiación difusa y global.

Raíz del error cuadrático medio Coeficiente de correlación

\begin{tabular}{lcc}
\hline Radiación difusa & $1,84 \mathrm{E}+02$ & 0,65 \\
Radiación global & $2,55 \mathrm{E}+02$ & 0,75
\end{tabular}

Finalmente, en la table 4 se determina la raíz del error cuadrático medio entre las series y la correlación de los datos, valores que comparados con las magnitudes y variación que se da en los datos resultan razonables, dado que son series de datos no lineales con un alto grado de caoticidad.

\section{Conclusiones.}

- El estudio permitió conocer la variación de la radiación solar en la zona de Urbina, la cual se caracteriza por ser altamente caótica (10 coeficientes positivos de Lyapunov),

- Los niveles de radiación difusa y global en la zona de Urbina supera las 8 horas de radiación efectiva; resultado que, complementado con la altura y sus niveles medidos, le da un alto potencial de energía solar.

- Las radiaciones difusas y globales predichas no presentan diferencias significativas al $95 \%$, según la prueba Boostrap, respecto a las reales, y a pesar de sus características dinámicas, presentan correlación alta entre las series, y la raíz del error cuadrático medio, respecto a su rango de variación se puede considerar razonable. 
- Los resultados de la investigación permitirán aprovechar estos recursos de mejor manera, tanto para la generación de energía, como para la agroindustria, lo cual es importante, considerando que es una de las zonas más productivas del país.

\section{Agradecimiento.}

Al Proyecto de Monitoreo Hídrico en la Provincia de Chimborazo del Centro de Energías Alternativas y Ambiente (CEAA) y al Instituto Nacional de Meteorología e Hidrología (INAMHI) por su colaboración en el desarrollo del artículo y la información suministrada.

\section{Referencias bibliográficas.}

Cañada J, Salvador D., (1997), Radiación solar, Valencia España, ed. Servicio de publicaciones de la Universidad Politécnica de Valencia, , Pg, 29-39, 47-63.

Constantin y, Foias C, (2006), Global Lyapunov exponents, Kaplan-Yorke formulas and the dimension of the attractors for 2D navier-stokes equations, Communications on Pure and Applied Mathematics, pp. 1-27.

Constantin y, Foias C., (2006), Global Lyapunov exponents, Kaplan-Yorke formulas and the dimension of the attractors for 2D navier-stokes equations", Communications on Pure and Applied Mathematics, pp. 1-27.

Domański P.D., Ławryńczuk M, (2020.) Control Quality Assessment of Nonlinear Model Predictive Control Using Fractal and Entropy Measures. In: Lacarbonara W., Balachandran B., Ma J., Tenreiro Machado J., Stepan G. (eds) Nonlinear Dynamics and Control. Springer, Cham.

Escudero A., Haro S., (2017), Suavizado de curvas mediante B-spline para el análisis funcional de la radiación solar global, Revista Perfiles, pp 35-43.

Fernández, D., (2014). Reducción del ruido y predicción de series temporales de alta frecuencia mediante sistemas dinámicos no lineales y técnicas neurales. Banco Nacional de Uruguay. ISSN 1688-7565, https://www.bcu.gub.uy/Estadisticas-eIndicadores/Documentos\%20de\%20Trabajo/1.2014.pdf

Gallego J., Aplicación de la teoría de caos para el análisis y pronóstico de series de tiempo financieras en Colombia, [Tesis Maestría], Colombia, UNC, 2010.

Garín F. Janampa A., Juan M. Pesantes R. y Martín B. Sandoval C., 2015, Generalization of the kolmogorov-sinai entropy: z-logistic maps, Anales Científicos, 76, pp. 237-240. 
Haro A., Llosas Y. Limaico C,. (2016), Predicción de datos meteorológicos en cortos intervalos de tiempo en la ciudad de Riobamba usando la teoría del caos, Revista Iberoamericana de Sistemas, Cibernética e Informática, pp. 35-4, 2016.

Hegger, R., Kantz, H., Thomas Schreiber, The TISEAN software package, (2007), [Consulta: 11 diciembre 2019]. Disponible en: https://aip.scitation.org/doi/abs/10.1063/1.166424.

Ivancevic V.G., Ivancevic T.T. , (2007). Introduction to Attractors and Chaos. In: HighDimensional Chaotic and Attractor Systems. Intelligent Systems, Control and Automation: Science and Engineering, vol 32. Springer, Dordrecht.

Kantz H. and Schreiber T., Nonlinear Time Series Analysis, 2nd edition, Cambridge University Press, Cambridge, Inglaterra, 2004.

Martins O., Sadeeq M., Ahaneku I., (2011), Nonlinear Deterministic Chaos in Benue River Flow Daily Time Sequence, Journal of Water Resource and Protection, pp. 747-757.

Medvinsky, A.B., Nurieva, N.I., Rusakov, A.V. et al., (2017). Deterministic chaos and the problem of predictability in population dynamics. BIOPHYSICS 62, 92-108. https://doi.org/10.1134/S0006350917010122.

Pareja M. A,. Radiación solar y su aprovechamiento energético, Barcelona España, Marcombo S.A.; 2010.

Puente, V. C., (2018): Estadística descriptiva e inferencial. Retrieved from https://ebookcentral.proquest.com.

Reyes S., Introducción a la meteorología, Universidad de Baja California, 2001, Pg. 3050

Ruelle D., Early chaos theory, Physics Today, May, 2013, pp. 27.

Sauer T., Yorke J., Casdagli M. (1991), Attractor Reconstruction and Control Using Interspike Intervals, Department of Mathematics, George Mason University, USA, J. Stat. Phys., Pp. 565-579.

Taher A. zar and Vaidyanathan S., (2016), Advances in Chaos Theory and Intelligent Control, Springer International Publishing Switzerland.

Zuñiga, L., Paguay M., Haro, et al, (2017): Bandas de confianza bootstrap en regresión polinómica. Revista Perfiles, 17, 12-18

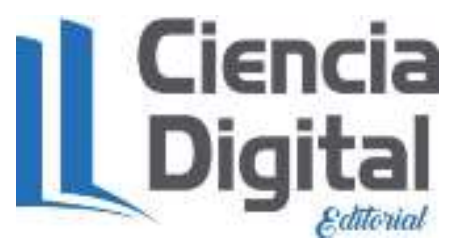




\section{PARA CITAR EL ARTÍCULO INDEXADO.}

Perugachi Cahueñas, N. P., Lara Sinaluisa, J. M., \& Haro Velasteguí, A. X. (2021). Análisis de la variación y predicción de radiación solar en la zona de Urbina, usando la teoría del caos. AlfaPublicaciones, 3(3.1), 341-356. https://doi.org/10.33262/ap.v3i3.1.101

\section{Ciencia Ligital

El artículo que se publica es de exclusiva responsabilidad de los autores y no necesariamente reflejan el pensamiento de la Revista Alfa Publicaciones.

El artículo queda en propiedad de la revista y, por tanto, su publicación parcial y/o total en otro medio tiene que ser autorizado por el director de la Revista Alfa Publicaciones.
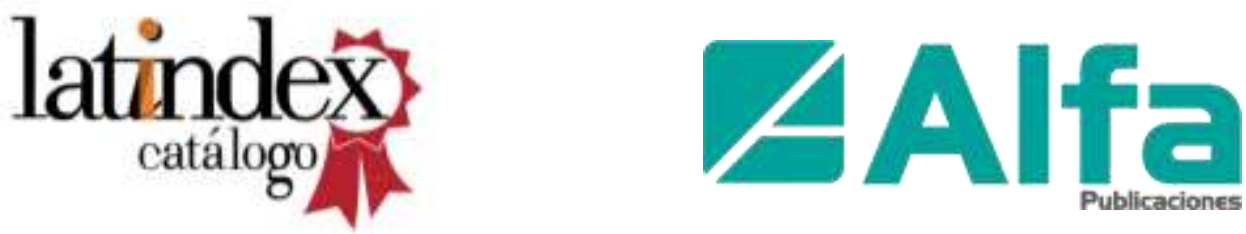